Mathematical Sciences Research Institute Publications

Editors

S.S. Chern

I. Kaplansky

C.C. Moore

I.M. Singer 


\section{Coxeter Graphs and Towers of Algebras}

Frederick M. Goodman Pierre de la Harpe Vaughan F. R. Jones

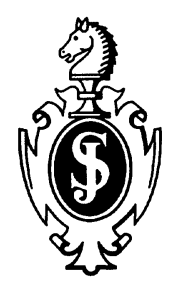

Springer-Verlag

New York Berlin Heidelberg

London Paris Tokyo 
Frederick M. Goodman

Department of Mathematics

University of Iowa

Iowa City, Iowa 52240

USA

Vaughan F.R. Jones

Department of Mathematics

University of California - Berkeley

Berkeley, California 94720

USA

Mathematical Sciences Research Institute

1000 Centennial Drive

Berkeley, California 94720

USA
Pierre de la Harpe

Section de Mathématiques

Université de Genève

CH-1211 Genève 24

Switzerland

Mathematical Subject Classification (1980): 46L10, 05C50, 16A40

Library of Congress Cataloging-in-Publication Data

Goodman, Frederick M.

Coxeter graphs and towers of algebras.

(Mathematical Sciences Research Institute publications ; 14)

Bibliography: p.

1. Class field towers. 2. Coxeter graphs.

I. La Harpe, Pierre de. II. Jones, Vaughan F.R.,

1952- . III. Title. IV. Series.

QA247.G68 $1989 \quad 512^{\prime} .55 \quad 89-5991$

Printed on acid-free paper.

(C) 1989 by Springer-Verlag New York Inc.

Softcover reprint of the hardcover 1st edition 1989

All rights reserved. This work may not be translated or copied in whole or in part without the written permission of the publisher (Springer-Verlag, 175 Fifth Avenue, New York, NY 10010, USA), except for brief excerpts in connection with reviews or scholarly analysis. Use in connection with any form of information storage and retrieval, electronic adaptation, computer software, or by similar or dissimilar methodology now known or hereafter developed is forbidden.

The use of general descriptive names, trade names, trademarks, etc. in this publication, even if the former are not especially identified, is not to be taken as a sign that such names, as understood by the Trade Marks and Merchandise Marks Act, may accordingly be used freely by anyone.

Camera-ready copy prepared by the authors. 


\section{PREFACE}

The paper [Jo1] on subfactors of von Neumann factors has stimulated much research in von Neumann algebras. Quite unexpectedly, it was discovered soon after the appearance of [Jo1] that certain algebras which are used there for the analysis of subfactors could also be used to define a new polynomial invariant for links [Jo3]. The period of activity following this discovery saw the creation of a number of related invariants as well as the successful use of these invariants in knot theory. Furthermore, recent effort to understand the fundamental nature of the the new link invariants has led to connections with invariant theory, statistical mechanics and quantum field theory. In turn the link invariants, the notion of a quantum group, and the quantum Yang-Baxter equation have had great impact on the study of subfactors.

It is not yet the time to give a comprehensive account of these developments, and we make no attempt to do so here. Our subject is certain algebraic and von Neumann algebraic topics closely related to the original paper [Jo1]. However, in order to promote, in a modest way, the contact between diverse fields of mathematics, we have tried to make this work accessible to the broadest audience. Consequently, this book contains much elementary expository material.

We give here a brief preview of the book. Each of the four chapters has its own introduction, with a more thorough description of the contents.

Chapter 1 begins with a (slightly new guise of) a familiar combinatorial problem: to classify finite matrices over the non-negative integers which have Euclidean norm no greater than 2. These are classified by the ubiquitous Coxeter graphs of type A, D, or E (see [HHSV] for other occurrences of these graphs) and the set of possible norms is $\{2\} \cup\{2 \cos \pi / q: q \geq 2\}$.

The central theme of the book - the discussion of which begins in Chapter $2-$ is the tower of algebras $M_{0} \subset M_{1} \subset \cdots \subset M_{k} \subset \cdots$ determined by a pair $M_{0} \subset M_{1}$ of algebras (with the same identity). The tower can be used to define various invariants of the pair, including the index $\left[\mathrm{M}_{1}: \mathrm{M}_{0}\right]$

In Chapters 2 and 3, we study two cases of the tower construction in detail.

In Chapter 2, the algebras are finite direct sums of full matrix algebras over some field. A pair $M_{0} \subset M_{1}$ is described, up to isomorphism, by an inclusion matrix $\Lambda$ with non-negative integer entries. This matrix may be encoded as a graph, known as the Bratteli diagram of the pair. It turns out that the index $\left[\mathrm{M}_{1}: \mathrm{M}_{0}\right]$ equals $\|\Lambda\|^{2}$; thus it follows from Chapter 1 that $\left[\mathrm{M}_{1}: \mathrm{M}_{0}\right] \leq 4$ if and only if the Bratteli diagram is a Coxeter graph of type A, D, or E. 
In Chapter 3, the algebras are finite von Neumann algebras with finite dimensional centers. Somewhat surprisingly, the results of Chapter 2 essentially extend to this setting. But now a pair $M_{0} \subset M_{1}$ is (partially) described by an inclusion matrix $\Lambda$ with entries in $\{2 \cos \pi / q: q \geq 2\} \cup\{r: r \geq 2\}$, and pairs with index no greater than 4 are associated to Coxeter graphs of arbitrary type, including types B,F,G,H,I.

Finally, Chapter 4 is a further analysis of pairs N C M of finite factors of finite index. There are two main themes. The first is the notion of a commuting square, due to Popa [Pop1], and its use in approximating pairs of hyperfinite $\mathrm{II}_{1}$ factors simultaneously by finite dimensional von Neumann algebras. The second theme is the derived tower of a pair of $\mathrm{II}_{1}$ factors, which is the chain of (necessarily finite dimensional) relative commutants $M_{0}{ }^{\prime} \cap M_{k}$ in the tower. All the information in the derived tower can be encoded in a (possibly infinite) graph, the principal graph of the pair. When the index is less than 4 , the graph is a Coxeter graph of type A, D, or E.

In Chapter 2, we also describe how a certain quotients of the Hecke algebra of type A appear in the tower construction associated to a pair $\mathrm{M}_{0} \subset \mathrm{M}_{1}$ of, say, finite dimensional semi-simple algebras over the field C. For each choice of a positive faithful trace $\operatorname{tr}$ on $M_{1}$, there is a unique trace preserving conditional expectation $E_{1}: M_{1} \rightarrow M_{0}$, and it turns out that $M_{2}$ is naturally generated by $M_{1}$ and $E_{1}$. Now if the trace tr satisfies the so-called Markov condition, then the situation propagates up the tower, and each algebra $\mathrm{M}_{\mathrm{k}+1}$ is naturally generated by $\mathrm{M}_{\mathrm{k}}$ and a conditional expectation $E_{k}: M_{k} \rightarrow M_{k-1}$, for all $k \geq 1$. Moreover the $E_{k}$ 's are idempotents which satisfy the "braiding" relations

$$
\begin{array}{ll}
\beta E_{i} E_{j} E_{i}=E_{i} & \text { if }|i-j|=1, \text { and } \\
E_{i} E_{j}=E_{j} E_{i} & \text { if }|i-j| \geq 2,
\end{array}
$$

where $\beta=\left[\mathrm{M}_{1}: \mathrm{M}_{0}\right]$. The abstract algebra $\mathcal{A}_{\beta, \mathrm{k}}$ presented by generators $\left\{1, E_{1}, \cdots E_{k-1}\right\}$ and relations as above is a quotient of the Hecke algebra $H_{k}(q)$, where $q \in \mathbf{C}$ satisfies $\beta=2+q+q^{-1}$.

Although we do not discuss this in the text, we might remark here that the map

$$
\mathbf{B}_{\infty} \longrightarrow \mathrm{H}_{\infty}(\mathrm{q}) \longrightarrow \operatorname{alg}\left\{\mathbf{1}, \mathrm{E}_{1}, \cdots\right\} \stackrel{\operatorname{tr}}{\longrightarrow} \mathrm{C}
$$

where $\mathbf{B}_{\infty}$ is the inductive limit of the braid groups $\mathbf{B}_{\mathrm{k}}$, is, up to a normalization, the Jones link invariant [Jo3]. Also let us point out that to obtain the Jones invariant in this way, it is necessary to deal only with finite dimensional algebras, not the less familiar infinite dimensional von Neumann factors. 
There are several appendices.

Appendix I extends the computations of Chapter 1.

Appendix II.a relates complex semi-simple algebras and finite dimensional $\mathrm{C}^{*}$-algebras. Appendix II.b explains one appearance of the algebras $d_{\beta, \mathrm{k}}$ in statistical mechanics. Appendix II.c is a further discussion of $\boldsymbol{\lambda}_{\beta, \mathrm{k}}$ for special values of $\beta$.

Appendix III is an exposition of Hecke subgroups in $\mathrm{PSL}_{2}(\mathbb{R})$, and thus another famous occurrence of the sequence $(2 \cos (\pi / \mathrm{k}))_{\mathrm{k} \geq 3}$.

It is a pleasure to record our gratitude to numerous friends and colleagues for their generous help, including:

R. Baldi, D. Bichsel, H. Dherete, M. Kervaire, A. Ocneanu, M. Pimsner, S. Popa, G. Skandalis, C. Skau, R. Steinberg, V. Sunder, A. Valette, and H. Wenzl.

We gratefully acknowledge support from the MSRI in Berkeley, the IHES in Bures, the United States NSF, the IMA in Minneapolis, and our home institutions during our work on this project. 


\section{CONTENTS}

Preface

Chapter 1. Matrices over the natural numbers: values of the norm, classification, and variations.

1.1. Introduction.

1.2. Proof of Kronecker's theorem. 4

1.3. Decomposability and pseudo-equivalence. $\quad 8$

1.4. Graphs with norms no larger than 2. 13

1.5. The set $\mathbf{E}$ of norms of graphs and integral matrices. 24

Chapter 2. Towers of multi-matrix algebras. $\quad 28$

$\begin{array}{ll}2.1 . & \text { Introduction. } \\ 28\end{array}$

2.2. Commutant and bicommutant 36

2.3. Inclusion matrix and Bratteli diagram for inclusions of multi-matrix algebras. $\quad 42$

2.4. The fundamental construction and towers for multi-matrix algebras. $\quad 57$

2.5. Traces. 66

2.6. Conditional expectations. $\quad 69$

2.7. Markov traces on pairs of multi-matrix algebras. $\quad 80$

2.8. The algebras $\mathcal{A}_{\beta, \mathrm{k}}$ for generic $\beta$. 86

2.9. An approach to the non-generic case. 102

2.10. A digression on Hecke algebras. 112

2.10.a. The complex Hecke algebra defined by $\mathrm{GL}_{n}(\mathrm{q})$ and its Borel

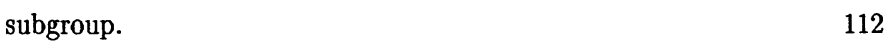

2.10.b. The Hecke algebras $\mathrm{H}_{\mathrm{q}, \mathrm{n}^{\circ}}$. 116

2.10.c. Complex representations of the symmetric group. 118

2.10.d. Irreducible representations of $\mathrm{H}_{\mathrm{q}, \mathrm{n}}$ for $\mathrm{q} \notin \Omega$. 119

2.11. The relationship between $\mathcal{A}_{\beta, \mathrm{n}}$ and the Hecke algebras. 123

Chapter 3. Finite von Neumann algebras with finite dimensional centers. $\quad 128$

$\begin{array}{ll}3.1 . & \text { Introduction. } \\ 3.2 & 128\end{array}$

3.2. The coupling constant: definition. 133

3.3. The coupling constant: examples. 142

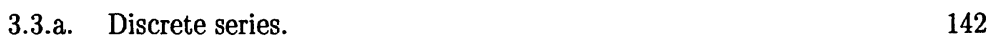

3.3.b. Factors defined by icc groups. 143

3.3.c. $\mathrm{W}^{*}(\Gamma)$-modules associated to subrepresentations of $\lambda_{\mathrm{G}}$. 144 
3.3.d. The formula $\operatorname{dim}_{\Gamma}(\mathrm{H})=\operatorname{covol}(\Gamma) \mathrm{d}_{\pi}$.

3.3.e. A digression on the Peterson inner product.

3.4. Index for subfactors of $\mathrm{II}_{1}$ factors.

3.5. Inclusions of finite von Neumann algebras with finite dimensional centers

3.6. The fundamental construction.

3.7. Markov traces on $\operatorname{End}_{\mathrm{N}}(\mathrm{M})$, a generalization of index.

Chapter 4. Commuting squares, subfactors, and the derived tower.

4.1. Introduction.

4.2. Commuting squares.

4.3. Wenzl' $\mathrm{s}$ index formula.

4.4. Examples of irreducible pairs of factors of index less than 4, and a lemma of C. Skau.

4.5. More examples of irreducible paris of factors, and the index value $3+3^{1 / 2}$.

4.6. The derived tower and the Coxeter invariant. 212

$\begin{array}{ll}\text { 4.7. Examples of derived towers } & 219\end{array}$

$\begin{array}{ll}\text { 4.7.a. Finite group actions. } & 219\end{array}$

4.7.b. The $A_{n}$ Coxeter graphs. 220

4.7.c. A general method. 221

4.7.d. Some examples of derived towers for index 4 subfactors. 222

4.7.e. The tunnel construction. 224

4.7.f. The derived tower for $R \supset R_{\beta}$ when $\beta>4$. 225

Appendix I. Classification of Coxeter graphs with spectral radius just beyond the Kronecker range. 232

I.1. The results. 232

I.2. Computations of characteristic polynomials for ordinary graphs. 235

I.3. Proofs of theorems I.1.2 and I.1.3. 243

Appendix II.a. Complex semisimple algebras and finite dimensional $\mathrm{C}^{*}$-algebras 253

Appendix II.b. The algebras $\lambda_{\beta, \mathrm{k}}$ in statistical mechanics. 259

Appendix II.c. More on the algebras $\mathcal{A}_{\beta, \mathrm{k}}$ for non-generic $\beta$. 266

Appendix III. Hecke groups and other subgroups of $\operatorname{PSL}(2, \mathbb{R})$ generated by parabolic pairs. $\quad 274$

$\begin{array}{ll}\text { References. } & 281\end{array}$

$\begin{array}{ll}\text { Index. } & 287\end{array}$ 